\title{
Brainstem Auditory Function at Term in Preterm Babies With and Without Perinatal Complications
}

\author{
ZE D. JIANG, DOROTHEA M. BROSI, ZHI HAU LI, CHAO CHEN, AND ANDREW R. WILKINSON \\ Children's Hospital [Z.D.J., Z.H.L., C.C.], Fudan University, Shanghai 200032, China; Neonatal Unit \\ [Z.D.J., D.M.B., A.R.W.], Department of Paediatrics, University of Oxford, John Radcliffe Hospital, \\ Oxford OX3 9DU, United Kingdom
}

\begin{abstract}
ABST
Functional integrity of the auditory brainstem was studied at
term in babies born at 30-36 wk of gestation using maximum
length sequence brainstem auditory evoked response (MLS
BAER). Compared with normal term babies, preterm babies who
had perinatal complications showed a tendency of an increase in
wave V latency and I-V and III-V intervals at all 91-910/s
clicks, with statistical significance at higher rates. Wave V
latency and I-V interval increased significantly at $455 / \mathrm{s}$ and
910/s. III-V interval increased at all click rates, which was more
significant at higher rates. III-V/I-III interval ratio increased at
most rates. Waves III and V amplitudes reduced significantly
mainly at $455 / \mathrm{s}$ and 910/s. In preterm babies who had no
perinatal complications, there were no major MLS BAER abnor-
malities except an increase in III-V interval at 91-910/s. By
comparison, the preterm babies with perinatal complications had
\end{abstract}
a significant increase in wave V latency, I-V and III-V intervals, and III-V/I-III interval ratio at 455/s and 910/s clicks. These results suggest that although there are no major abnormalities in brainstem auditory function in preterm babies without perinatal complications, the auditory brainstem, mainly the more central part, in preterm babies with perinatal complications is impaired, which becomes more apparent at very high stimulus rates. We conclude that preterm babies with perinatal complications are at high risk of central auditory impairment. (Pediatr Res 58: 11641169, 2005)

Abbreviations
BAER, brainstem auditory evoked response
dB nHL, decibel normal hearing level
MLS, maximum length sequence

The integrity of auditory system is the base for language and speech development and has a tremendous impact on cognitive development. Auditory impairment, if undetected, will impede speech, language, and cognitive development $(1,2)$. Impairment of the central auditory system eliminates or modulates afferent activity in the peripheral auditory system and then changes the structure and function of the developing brain that is highly plastic, leading to neurodevelopmental deficits. Preterm babies in the neonatal intensive care unit often have various perinatal complications and/or major conditions that may directly or indirectly impair the central nervous system (CNS), including the central auditory system. Therefore, these babies may be at high risk of central auditory impairment, although this remains to be determined. Research suggests that central auditory impairment in children born with perinatal problems occurs more frequently than commonly recognized

Received January 3, 2005; accepted May 4, 2005.

Correspondence: Ze Dong Jiang, M.D., Ph.D., Neonatal Unit, Department of Paediatrics, University of Oxford, John Radcliffe Hospital, Headington, Oxford OX3 9DU, U.K.: e-mail: zedong.jiang@paediatrics.ox.ac.uk

Supported by Defeating Deafness, Oxfordshire Health Services Research Committee, and WellChild, U.K.

DOI: $10.1203 / 01 . p d r .0000183783 .99717 .2 b$
$(3,4)$. However, detection of the impairment in infants is difficult.

There are numerous reports on auditory impairment in preterm babies or children born preterm $(5,6)$. These reports generally concentrated on peripheral auditory impairment and showed that peripheral impairment, including conductive and sensory or sensorineural, is a common abnormality present in preterm babies. On the other hand, there are very few reports on central auditory impairment.

As an objective test to study functional integrity of the auditory brainstem, the BAER has been used to study functional development of the human auditory system and detect auditory impairment and neuropathology that affects the brainstem auditory pathway (7-12). Nevertheless, the BAER, recorded using conventional averaging technique, has a relatively high false-negative rate.

To improve early detection of central auditory abnormalities, we recently used the MLS to study BAER. This technique can present acoustic stimuli much higher than possible with a conventional averaging technique (13-16). Our results demonstrate that the MLS BAER can improve the detection of neuropathology that affects the auditory brainstem, typically hypoxia-ischemia $(15,16)$. The major goal of the reported study 
was to use MLS BAER to detect any brainstem auditory impairment in preterm babies, particularly those who have perinatal complications and/or major conditions that may directly or indirectly affect the CNS.

\section{METHODS}

Subjects. The following three groups of subjects were recruited.

The first group was the preterm high-risk group (babies who had perinatal complications and/or major conditions that may directly or indirectly affect the CNS): Seventy-three preterm babies who had at least one of the following perinatal complications or major conditions (some babies had more than one complication or condition): grade III to IV intraventricular hemorrhage ( $n=$ 16), periventricular leukomalacia $(n=19)$, Apgar scores $\leq 6$ at 1 and/or 5 min with or without hypoxic-ischemic encephalopathy $(n=34)$, hyperbilirubinemia at a serum level requiring exchange transfusion $(n=9)$, bacterial meningitis $(n=2)$, severe respiratory distress syndrome $(n=19)$, pneumonia $(n=22)$, and/or requiring artificial ventilation lasting $5 \mathrm{~d}$ or longer $(n=15)$. Babies who have severe intrauterine growth retardation (birth weight below the third percentile) were excluded to avoid the confounding effect on the BAER (17). Gestation ranged from 30-36 wk $(32.5 \pm 2.5 \mathrm{wk})$ and birth weight from 1150 to $3300 \mathrm{~g}(1800 \pm 508 \mathrm{~g})$.

The second was the preterm low-risk group (babies who had no perinatal complications and/or major conditions). Thirty-seven preterm babies who did not have any of the above perinatal complications and/or major conditions. Gestation ranged from $30-35 \mathrm{wk}(32.7 \pm 1.8 \mathrm{wk})$ and birth weight from 1331 to $2865 \mathrm{~g}(1868 \pm 419 \mathrm{~g})$, which did not differ significantly from the preterm high-risk group.

The third group was the term normal group. Thirty-eight healthy newborn babies served as term controls. None had any perinatal complications and/or major conditions, with a gestation of 37-41 wk $(39.0 \pm 1.3 \mathrm{wk}$ and birth weight 2633-4539 g (3507 $\pm 496 \mathrm{~g})$. Monaural BAER thresholds, determined by conventional BAER at 21/s click, were all $<20 \mathrm{~dB}$ nHL (normal hearing level) at the time of testing. There was no significant difference between the three groups of subjects in postconceptional age at which MLS BAER testing was undertaken.

The preterm babies were recruited from the Neonatal Unit, Department of Paediatrics, John Radcliffe Hospital, University of Oxford $(n=59$, including 39 high-risk and 20 low-risk babies) and from the Neonatal Unit, Children's Hospital of Fudan University, Shanghai ( $n=51$, including 34 high-risk and 17 low-risk babies). The term babies were recruited from the maternity words, Department of Obstetrics and Gynaecology, John Radcliffe Hospital, University of Oxford. At the time of MLS BAER testing (37-42 wk postconceptional age for preterm babies and 1-3 d after birth for term babies), all babies were in a stable clinical condition.

Recording and analysis of MLS BAER. A Bravo Portable Evoked Potential System (Nicolet Biomedical Inc. Madison, WI) was used to study MLS BAER. The recording started after the baby fell asleep naturally, often after a feed, and lay supine in a cot. No sedatives were used. Three gold-plated disk electrodes were placed at the middle forehead (positive), the ipsilateral earlobe (negative), and the contralateral earlobe (ground), respectively. Interelectrode impedances were maintained at $<10 \mathrm{k} \Omega$, often $5 \mathrm{k} \Omega$. To save the time of recording MLS BAER and to keep the recording and analyzing conditions consistent, only the left ear was tested in all subjects.

The MLS technique uses a patterned stimulus presentation rather than the uniformly spaced stimuli used in conventional BAER testing. Different patterned sequences of stimuli are created by omitting a portion (e.g. 50\%) of the stimuli in a pseudorandom fashion. Mathematically, a maximum length sequence is a quasirandom binary sequence represented by a train of $+1 \mathrm{~s}$ and $-1 \mathrm{~s}$. In its audiological application, it may be presented by $+1 \mathrm{~s}$ and $0 \mathrm{~s}$ or by clicks and silences. This stimulus consists of distinct pulses of uniform polarity and amplitude occurring at pseudorandom time intervals. Each pulse sequence is actually a series of pulses. Therefore, the accepted value and the number entered in the sweep count represent the number of sequences, not the number of discrete pulses as in conventional BAER. When there are $50 \%$ gaps in the MLS stimulus patterns, actual repetition rate fluctuates over time and the average rates are actually one half of the rates.

The nature of the stimulus and the newly developed processing technique makes it unnecessary to wait for the response of each pulse to be completed before applying a new pulse. Thus, the pulses can be delivered at maximum rates of up to 1000/s or even higher. Because the patterned sequences of stimuli are generated by the averaging computer, this information is then used to perform on-line deconvolution (separation, alignment, and averaging) of overlapping individual responses. As in recording of conventional BAER, each waveform of the response is filtered and the waveforms are averaged. The final
MLS BAER is then obtained by mathematically cross-correlating the collected data with a recovery sequence.

As reported previously $(15,16)$, the acoustic stimuli we used were rarefaction clicks of $100 \mu \mathrm{s}$, delivered monaurally through a TDH 39 earphone. Before recording MLS BAER, conventional BAER was recorded with clicks at 21/s for comparison and determining BAER threshold. The MLS BAER was elicited with clicks at the repetition rates 91/s, 227/s, 455/s, and 910/s in the first run. A reverse sequence was used in the second run. Click intensity was $60 \mathrm{~dB}$ nHL for all babies. In those with BAER thresholds, $>20 \mathrm{~dB}$ nHL higher intensities were also used so that the data of BAER central components can be compared between groups at the same hearing level (i.e. $\geq 40 \mathrm{~dB}$ above their thresholds).

The evoked brain responses were amplified and filtered at $100-3000 \mathrm{~Hz}$. If the data exceeded $91 \%$ of the sensitivity parameter setting $(51 \mu \mathrm{V})$, that sweep (artifact) was automatically rejected by the system. Sampling was manually discontinued whenever there were excessive muscle artifacts on the monitoring oscilloscope. Brain responses to 1500 trains of clicks were averaged for each run. Duplicate recordings were made in response to each stimulus condition to examine reproducibility.

The study protocol and procedures were approved by the Central Oxford Research Ethics Committee and the Children's Hospital Ethics Committee of Fudan University. Informed consent of parents and the pediatrician in charge was obtained for all subjects.

Data analysis. Measurement of wave latencies and amplitudes in MLS BAER recordings was carried out blindly to the medical history and clinical data of each subject. Wave latency (I, III, and V) was measured from the onset of click stimuli to the peak of a wave. Interpeak interval (I-V, I-III, and III-V) was calculated as the time between the peaks of any two waves. Wave I amplitude was measured from the peak of wave I to the lowest trough between waves I and III, and wave III amplitude was from the trough to the peak of wave III. Measurement of wave V amplitude was made from the peak of wave $\mathrm{V}$ to the following trough.

Wave latencies and amplitudes and interpeak intervals were measured and analyzed at a click intensity $\geq 40 \mathrm{~dB}$ above the threshold of each subject, i.e. $60 \mathrm{~dB}$ nHL for thresholds $\leq 20 \mathrm{~dB}$ nHL, $70 \mathrm{~dB}$ nHL for thresholds $>20-30 \mathrm{~dB}$ $\mathrm{nHL}$, or $80 \mathrm{~dB} \mathrm{nHL}$ for thresholds $>30 \mathrm{~dB} \mathrm{nHL}(15,16)$. However, to minimize the effect of peripheral auditory impairment on MLS BAER central components, babies with a significantly elevated BAER threshold $(>35 \mathrm{~dB}$ nHL), suggesting peripheral auditory disorders, were excluded.

The measurements of two replicated BAER recordings to each stimulus condition were averaged for data analyses. The mean and SD of each BAER variable at each stimulus condition were compared between groups using the analysis of variance (ANOVA) with a SPSS package (version 12). The correlation between BAER variables and click rate was also analyzed.

\section{RESULTS}

Similar to those in the term normal group, the latencies and interpeak intervals of all MLS BAER components in the preterm groups correlated positively with the repetition rate of clicks, i.e. increased with the increase in click rate $(r=$ 0.421-0.795, all $p<0.01$ ), while all wave amplitudes were correlated negatively with click rate, i.e. reduced with the increase in click rate $(r=-0.682$ to 0.751 , all $p<0.01)$. The III-V/I-III interval ratio in the two preterm groups correlated positively with click rate $(r=0.513$ and 0.414 , respectively, $p$ $<0.01)$. The change in the III-V interval with the change in click rate was more significant in the preterm high-risk group than in the preterm low-risk group and term group $(p<0.05)$. No correlation was found between V/I and V/III amplitude ratios and click rate in any of the three groups of subjects.

Comparison among preterm high-and low-risk groups and term group. In the preterm high-risk group, wave $\mathrm{V}$ latency and I-V and III-V intervals tended to increase and wave amplitudes tended to decrease at almost all click rates, compared with those in the preterm low-risk group and term group.

No statistically significant differences were found among the three groups in wave I and III latencies at any repetition rates of clicks. In wave V latency, however, the three groups of 
babies differed significantly at $455 / \mathrm{s}$ and $910 / \mathrm{s}$ clicks $(p<0.05$ and 0.001 , respectively, Fig. 1). The I-V interval also differed significantly at $455 / \mathrm{s}$ and $910 / \mathrm{s}(p<0.01$ and 0.001 , respectively, Fig. 2). The III-V interval differed significantly at all rates of clicks among the three groups and the difference increased with the increase in the click rate $(p<0.05-0.001$, Fig. 2). Similarly, the III-V/I-III interval ratio differed among the three groups, which was more significant at higher rates than at low rates $(p<0.05-0.001$, Fig. 3).

The amplitudes of waves III and V differed significantly among the three groups of babies at most click rates $(p<$ 0.05-0.001, Fig. 4). The differences were most significant at the very high rates of $455 / \mathrm{s}$ and $910 / \mathrm{s}$. Wave I amplitude differed at $21 / \mathrm{s}$ and $91 / \mathrm{s}$ clicks $(p<0.05$ and 0.05 ). Neither the V/I amplitude ratio nor the V/III amplitude ratio differed significantly among the three groups at any rates.

Comparison of preterm high-risk group and term group. No differences were found between the two groups in wave I and III latencies at any click rates. However, wave V latency and the I-V interval in preterm high-risk group tended to increase at all rates. The two groups differed significantly in wave V latency at $21 / \mathrm{s}, 455 / \mathrm{s}$, and $910 / \mathrm{s}(p<0.05,0.05$, and 0.001 , Fig. 1) and in I-V interval at 455/s and 910/s $(p<0.01$ and 0.001 , Fig. 2). III-V interval in preterm high-risk group increased significantly at all rates, particularly $455 / \mathrm{s}$ and $910 / \mathrm{s}$ $(p<0.05-0.001)$, while I-III interval did not differ significantly at any rates between the two groups (Fig. 2). III-V/I-III interval ratio in preterm high-risk group increased significantly at all click rates except 91/s ( $p<0.05-0.001$, Fig. 3).

Wave I amplitude in preterm high-risk group reduced at $21 / \mathrm{s}$ and $91 / \mathrm{s}$ ( $p<0.05$ and 0.05, Fig. 4). Wave III and V amplitudes in preterm high-risk group reduced with the increase in click rate more dramatically than in term group. Wave III amplitude reduced significantly at all rates in MLS BAER (91-910/s clicks, $p<0.05-0.01)$ and wave V amplitude reduced significantly at 455/s and 910/s $(p<0.01$ and 0.01 ). No differences were found between the two groups in $\mathrm{V} / \mathrm{I}$ and V/III amplitude ratios at any rates.

Comparison of preterm low-risk group and term group. No major differences were found between the two groups in the changes in MLS BAER variables with the change in click rate. In preterm low-risk group, none of the wave latencies differed significantly from the term group at any rates $(p<0.05$, Fig. $1)$. The I-V interval was similar to that in the term group at all rates (Fig. 2). However, the III-V interval increased significantly at all rates in MLS BAER (91-910/s, $p<0.05-0.01$, Fig. 2). On the other hand, the I-III interval decreased at most rates $(p<0.05$ at $21-455 / \mathrm{s})$. III-V/I-III interval ratio increased at all rates $(p<0.05-0.01$, Fig. 3).

All wave amplitudes in the preterm low-risk group were similar to those in the term group at all rates (Fig. 4). There were also no differences between the two groups in V/I or V/III amplitude ratios.

Comparison of preterm high- and low-risk groups. In the preterm high-risk group, all wave latencies tended to increase at all click rates, compared with those in the preterm low-risk group (Fig. 1). Wave V latency increased significantly at $455 / \mathrm{s}$ and 910/s $(p<0.05$ and 0.01), although wave I and III latencies did not show any significant differences from those in the preterm low-risk group at any rates. Similarly, all interpeak intervals in the preterm high-risk group tended to increase at all rates, but only the I-V and III-V intervals differed significantly from those in the preterm low-risk group at 455/s and 910/s ( $p$ $<0.05-0.001$, Fig. 2). The III-V/I-III interval ratio also increased significantly at the two rates $(p<0.05$ and 0.05 , Fig. $3)$.

Compared with the preterm low-risk group, the amplitudes of all MLS BAER waves in the preterm high-risk group tended to decrease (Fig. 4). Wave III amplitude reduced significantly at all rates except 227/s $(p<0.05-0.01)$. Wave V amplitude also decreased significantly at most rates $(21 / \mathrm{s}, 227 / \mathrm{s}, 455 / \mathrm{s}$, and 910/s, $p<0.05-0.01$ ). No statistically significant differences were found between the two groups in V/I and V/III amplitude ratios at any rates.

Figure 5 shows sample recordings of MLS BAER in subjects from each of the three subgroups. There are no apparent differences between the low-risk preterm baby (B, female, 33-wk gestation) and the normal term baby (A, female, 39-wk gestation) in any wave latencies and amplitudes and interpeak intervals. By comparison, the high-risk preterm baby $(\mathrm{C}$, female, 34-wk gestation, hypoxic-ischemic encephalopathy with periventricular leukomalacia) has a significant increase in wave V latency, I-V and particularly III-V intervals, and a significant reduction in wave $\mathrm{V}$ amplitude at 455 and 910/s clicks.
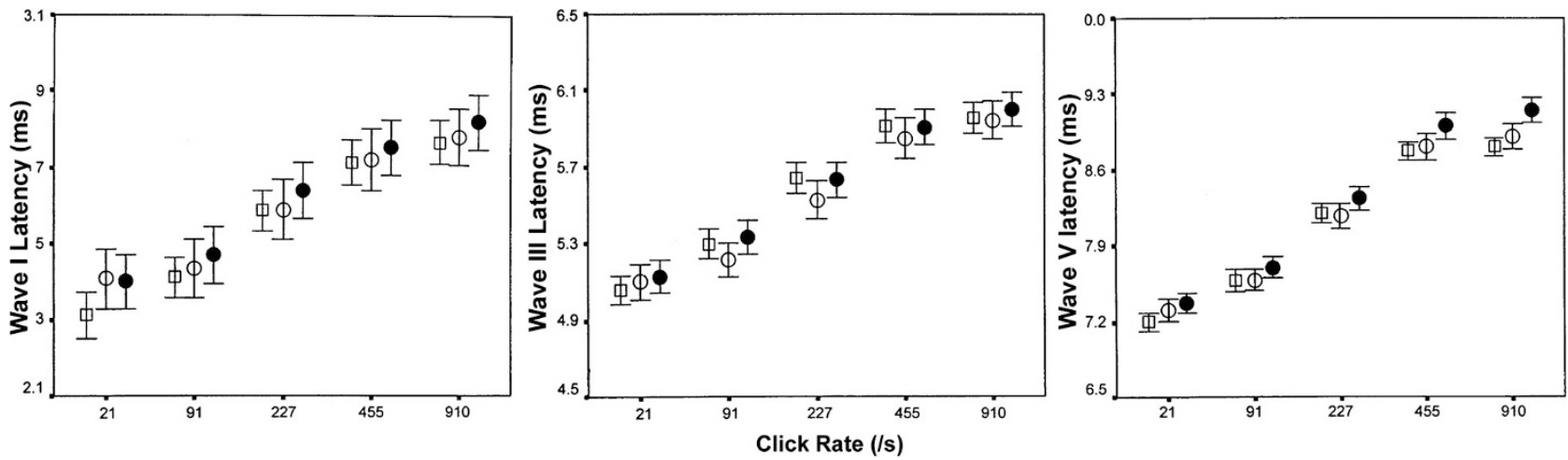

Figure 1. Wave latencies. Means and standard errors of wave I, III, and V latencies at different click rates in preterm babies [preterm low-risk $(O)$; preterm high-risk (๑)], recorded at term, and term controls $(\square)$. See text for $p$ values of statistical significance between groups. 

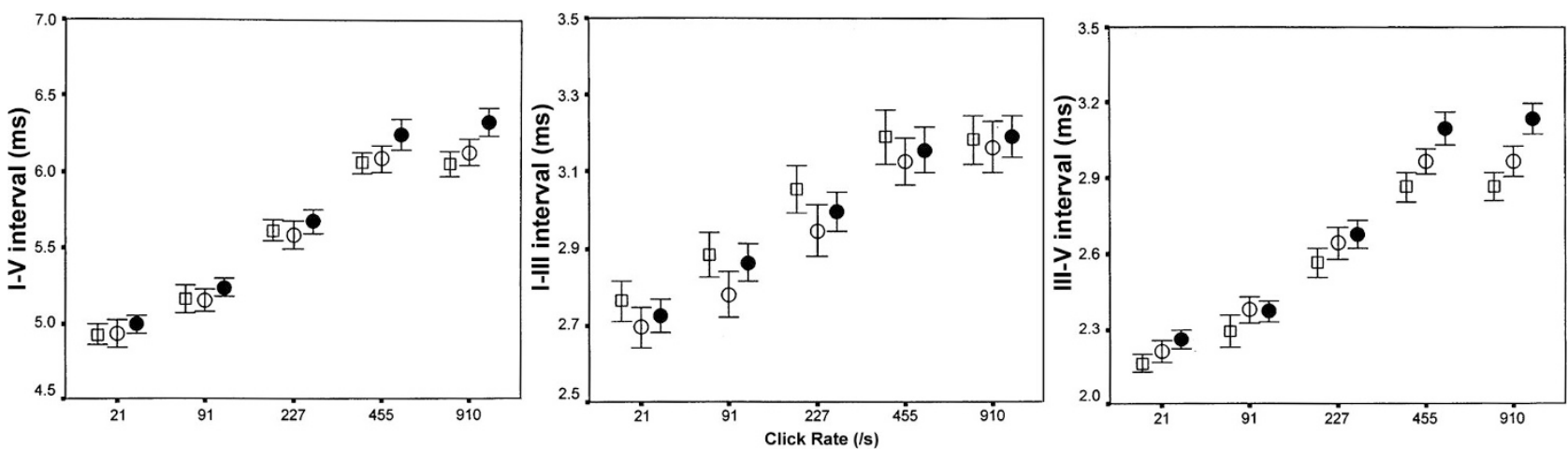

Figure 2. Interpeak intervals. Means and standard errors of I-V, I-III and III-V intervals at different click rates in preterm babies [preterm low-risk $(O)$; preterm high-risk (@)], recorded at term, and term controls ( $\square$ ). See text for $p$ values.

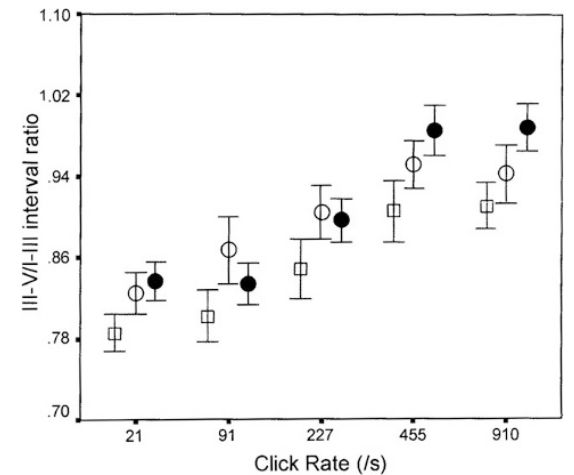

Figure 3. Interval ratio. Means and standard errors of the III-V/I-III interval ratio at different click rates in term and preterm babies [preterm low-risk $(\bigcirc)$; preterm high-risk (๑)], recorded at term $(\square)$ See text for $p$ values.

\section{DISCUSSION}

The present study found that MLS BAER variables that mainly reflect central auditory function (wave $\mathrm{V}$ latency and $\mathrm{I}-\mathrm{V}$ and III-V intervals) differed significantly among the three groups of babies. The differences, which occurred most significantly at very high click rates (455/s and 910/s), were predominantly produced by the significant increase in these MLS BAER variables in the preterm high-risk group. These results suggest that the auditory brainstem, mainly the more central part, in preterm babies who have perinatal complications is impaired and/or delayed in development, which becomes more evident following more stressful high-rate stimulation. On the other hand, there were no major MLS BAER abnormalities in the preterm low-risk group, suggesting no major abnormalities in central auditory function.

Preterm babies with perinatal complications are at highrisk of central auditory impairment. Previous follow-up studies showed that some of the children who were born preterm had various degrees of neurologic impairment and developed neurodevelopmental deficits, and these children were often associated with perinatal complications or problems (18). The present study revealed that at term the preterm babies with perinatal complications had clear abnormalities in MLS BAER, which occurred mainly at 455/s and 910/s. The main abnormalities included a significant increase in wave $\mathrm{V}$ latency, I-V interval, the so-called central conduction time, and
III-V interval, and a significant reduction in waves III and V amplitudes.

In BAER, I-III and III-V intervals, the two components of the I-V interval, generally reflect functional integrity of the peripheral and central parts of the auditory brainstem, respectively. In the present study, since I-III interval was relatively normal, the significant increase in wave $\mathrm{V}$ latency and the I-V interval in the preterm babies with perinatal complications must be essentially produced by the significant increase in the III-V interval. This is supported by the finding of a significant increase in III-V/I-III interval ratio. The present study also found a significant reduction in wave $\mathrm{V}$ amplitude at very high rate stimulation. These MLS BAER abnormalities indicate brainstem auditory impairment and/or developmental delay in the preterm babies with perinatal complications, which becomes more apparent following very stressful stimulation. In addition, the increased rate-dependent change in the III-V interval suggests that the efficacy of synaptic transmission in the central auditory system is impaired $(15,16)$.

It is known that preterm birth is associated with a number of obstetric, social, physical, and medical factors. In the majority of instances, however, it is difficult to identify accurately the major risk factors for neurologic impairment and developmental deficits. Many previous studies of peripheral auditory impairment in preterm babies or children born preterm found that there are often several potential causative factors for the impairment and no one cause can be identified $(1,5,6)$. The high incidence of peripheral auditory impairment in preterm babies has been linked to several risk factors: family history of hereditary childhood sensorineural hearing loss, in utero infection (e.g. cytomegalovirus, rubella, syphilis, herpes, and toxoplasmosis), craniofacial anomalies, very or extremely low birth weight, ototoxic medications, bacterial meningitis, low Apgar scores, mechanical ventilation lasting $5 \mathrm{~d}$ or longer, hyperbilirubinemia, ambient incubator noise, stigmata, or other findings associated with a syndrome known to include a sensorineural and/or conductive hearing loss $(1,5,6)$.

To identify accurately the risk factors for auditory impairment, a substantial number of subjects are needed so that each risk factor has a sufficient number of subjects for statistical analysis. In the reported study, we included a range of perinatal complications and/or major conditions that may put a baby at high risk of brain damage and/or auditory impairment. Due to 

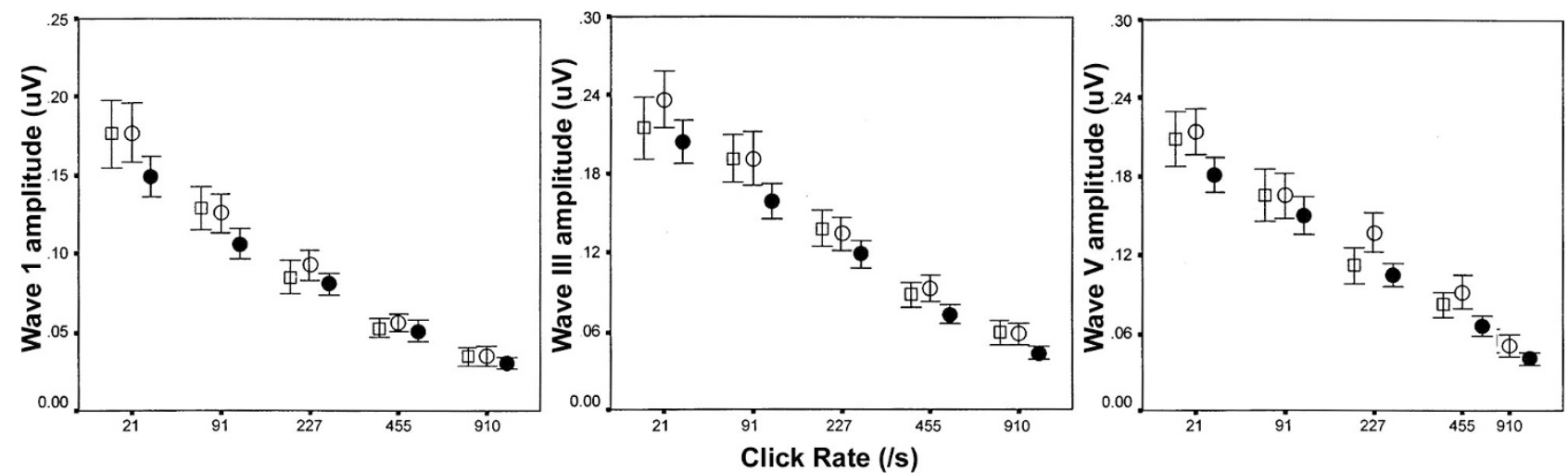

Figure 4. Wave amplitudes. Means and standard errors of wave I, III, and V amplitudes at different click rates in term and preterm babies [preterm low-risk $(\bigcirc)$; preterm high-risk $(\bullet)$ ], recorded at term $(\square)$ See text for $p$ values.
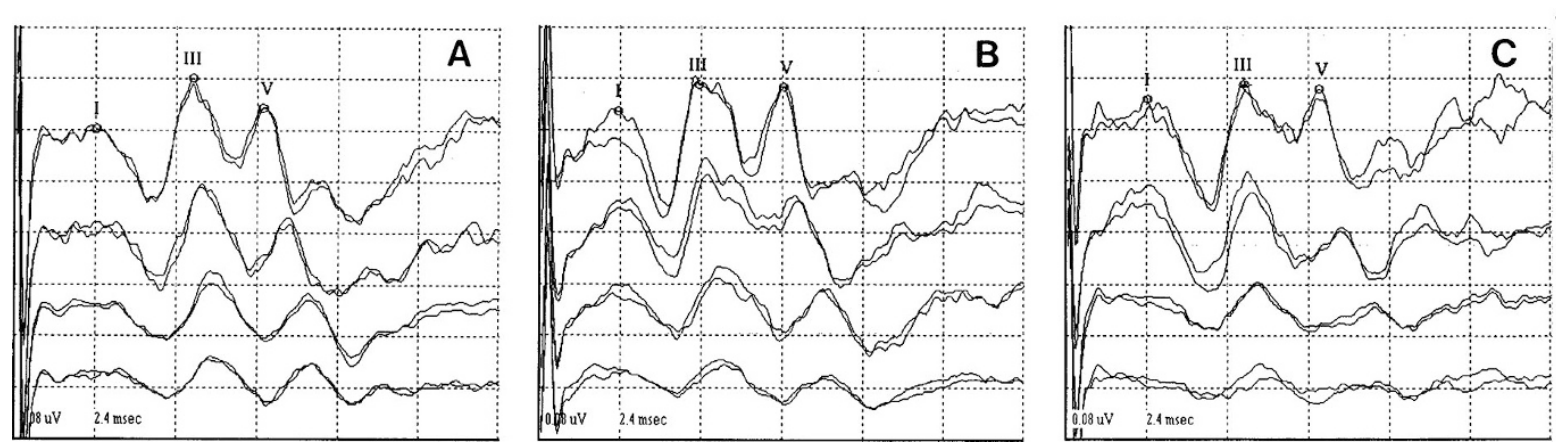

Click rate

(/sec)

91

227

455

910

Figure 5. Sample recordings of MLS BAER. (A) Normal term baby; $(B)$ preterm low-risk baby; and $(C)$ preterm high-risk baby. Compared with babies in $A$ and $B$, the baby in $C$ shows a significant increase in wave $\mathrm{V}$ latency, $\mathrm{I}-\mathrm{V}$ and particularly III-V intervals, and a significant reduction in wave $\mathrm{V}$ amplitude at 455 and $910 /$ s clicks.

the relatively small number of subjects in each complication or major condition, we did not carry out a detailed analysis of risk factors for MLS BAER abnormalities and identify the major risk factors for central auditory impairment. In addition, the clinical significance of the MLS abnormalities discovered in the present study needs to be further explored. We are now continuing to recruit new subjects to collect sufficient data for identifying major risk factors some time in the future, improving our understanding of the clinical significance of the MLS BAER abnormalities.

Preterm babies without perinatal complications are at low risk of central auditory impairment. Previous studies often found no abnormalities in central components of conventional BAER in preterm babies without perinatal problems $(8,10,12)$. In the present study, we did not find any major abnormalities in MLS BAER central components in the preterm babies without perinatal complications. The increase in wave $\mathrm{V}$ latencies and $\mathrm{I}-\mathrm{V}$ and III-V intervals was much less significant, compared with those in the preterm babies with complications. There was also no reduction in wave amplitudes at any click rates.

Nevertheless, the III-V interval in these preterm babies increased at higher click rates. On the other hand, the I-III interval decreased slightly at most rates. The increase in the III-V/I-III interval resulted from the increase in the III-V interval and the slight decrease in the I-III interval. These results suggest that brainstem auditory function is suboptimal in preterm babies without perinatal complications, which may be related to the less severe perinatal conditions associated with preterm birth.
Why was the I-III interval relatively normal in preterm babies with perinatal complications? Maturation of the BAER depends on both inborn and environmental factors. Sound experience in early life plays an important role in the development of the auditory system. Abnormal development may occur following exposure to abnormal acoustic environments. The slight decrease in the I-III interval in the preterm babies without perinatal complications is likely due to the preterm birth, which exposes the babies to sound environment ex utero earlier than term babies, leading to accelerated maturation or myelination in the more peripheral part of the auditory brainstem.

In the preterm babies who had perinatal complications, however, the I-III interval increased slightly, compared with that in the preterm babies without perinatal complications, and was similar to that in the term normal babies at all rates of clicks. As discussed above, preterm birth may accelerate the maturation of the auditory brainstem, leading to a decrease in the I-III interval. On the other hand, perinatal complications may impair the auditory brainstem or delay its development, resulting in an increase in I-III interval. In preterm babies with perinatal complications, the effect of maturational acceleration due to preterm birth offsets the effect of neural impairment or developmental delay due to perinatal complications. As a result, the I-III interval may not show any apparent change. It appears that the relatively normal I-III interval in the preterm babies with perinatal complications may not imply a completely normal function of the more peripheral part of the auditory brainstem. 
Very high rate stimulation while recording BAER improves the detection of central auditory abnormality. To improve the detection of neuropathology that affects the auditory brainstem, we used the method of increasing the rate of clicks while recording conventional BAER $(9,19,20)$. The results show that this method can improve the detection to a certain degree. An apparently normal or nearly normal BAER in response to routinely used low-rate stimulation may be abnormal when more stressful high-rate stimulation is used. However, there is a disadvantage in that BAER wave form morphology tends to deteriorate at rates higher than 51/s. This sometimes makes it difficult to measure BAER variables accurately and reliably. Furthermore, the conventional averaging technique can increase the repetition rate up to only about $100 / \mathrm{s}$. This limits the ability of increasing rate to improve detection of neuropathology in conventional BAER.

More recently, we have used MLS BAER to study functional integrity and maturation of the brainstem and central auditory system $(15,16)$. The major advantage of this technique is that it can present acoustic stimuli up to $1000 / \mathrm{s}$ or even higher. The much higher rates present a much stronger physiologic/temporal challenge to auditory neurons and permit a more exhaustive sampling of physiologic recovery than is possible using the relatively lower rates in conventional BAER (13-16). This stimulus of stress provides a potential to improve the detection of some neuropathology, particularly a subtle or early one, which may not be detected by presenting less stressful stimuli (i.e. low-rate stimulation) using a conventional averaging technique. Our recent studies in babies with perinatal hypoxia-ischemia have shown that MLS BAER can improve the detection of neuropathology that affects the auditory brainstem $(15,16)$.

Similar to our previous findings $(15,16)$, the present study showed that MLS BAER abnormalities usually increased with the increase in the rate of clicks and the abnormalities often occurred mainly at the very high rates of $455 / \mathrm{s}$ and $910 / \mathrm{s}$, which cannot be achieved in BAER using a conventional averaging technique. These findings indicate that the significant increase in click rate (e.g. 455/s and 910/s) can improve the detection of neuropathology that affect the auditory brainstem.

Acknowledgments. We thank the medical staff at the Neonatal Unit of the John Radcliffe Hospital, Oxford, and the
Children's Hospital of Fudan University, Shanghai, for their full cooperation and assistance in carrying out the research.

\section{REFERENCES}

1. Joint Committee on Infant Hearing, American Academy of Audiology, American Academy of Pediatrics, American Speech-Language-Hearing Association, Directors of Speech and Hearing Programs in State Health and Welfare Agencies 2000 Year 2000 position statement: principles and guidelines for early hearing detection and intervention programs. Joint Committee on Infant Hearing, American Academy of Audiology, American Academy of Pediatrics, American Speech-Language-Hearing Association, and Directors of Speech and Hearing Programs in State Health and Welfare Agencies. Pediatrics 106:798-817.

2. Yoshinaga-Itano C, Sedey AL, Coulter DK, Mehl AL 1998 Language of early- and later-identified children with hearing loss. Pediatrics 102:1161-1171

3. Doyle LW, Keir E, Kitchen WH, Ford GW, Rickards AL, Kelly EA 1992 Audiologic assessment of extremely low birth weight infants: a preliminary report. Pediatrics 90:744-749

4. Rance G, Beer DE, Cone-Wesson B, Shepherd RK, Dowell RC, King AM, Rickards FW, Clark GM 1999 Clinical findings for a group of infants and young children with auditory neuropathy. Ear Hear 20:238-252

5. Newton V 2001 Adverse perinatal conditions and the inner ear. Semin Neonatol 6:543-551

6. Meyer C, Witte J, Hildmann A, Hennecke KH, Schunck KU, Maul K, Franke U, Fahnenstich H, Rabe H, Rossi R, Hartmann S, Gortner L 1999 Neonatal screening for hearing disorders in infants at risk: incidence, risk factors and follow-up. Pediatrics 104:900-904.

7. Volpe JJ 2001 Specialized studies in the neurological evaluation. In: Volpe JJ (ed) Neurology of the Newborn, 4th ed. Philadelphia, WB Saunders, pp 134-177.

8. Jiang ZD 1995 Maturation of the auditory brainstem in low risk-preterm infants: a comparison with age-matched full term infants up to 6 years. Early Hum Dev 42:49-65

9. Jiang ZD, Brosi DM, Wilkinson AR 2002 Auditory neural responses to click stimuli of different rates in the brainstem of very preterm babies at term. Pediatr Res 51:454-459

10. Eggermont JJ, Salamy A 1988 Maturational time course for the ABR in preterm and full term infants. Hear Res 33:35-47

11. Pasman JW, Rotteveel JJ, de Graaf R, Stegeman DF, Visco YM 1992 The effect of preterm birth on brainstem, middle latency and cortical auditory evoked responses (BMC AERs). Early Hum Dev 31:113-129

12. Rotteveel JJ, de Graaf R, Colon EJ, Stegeman DF, Visco YM 1987 The maturation of the central auditory conduction in preterm infants until three months post term. II, The auditory brainstem responses (ABRs). Hear Res. 26:21-35

13. Lasky RE 1997 Rate and adaptation effects on the auditory evoked brainstem response in human newborns and adults. Hear Res 111:165-176

14. Jirsa RE 2001 Maximum length sequences-auditory brainstem responses from children with auditory processing disorders. J Am Acad Audiol 12:155-164

15. Jiang ZD, Brosi DM, Shao XM, Wilkinson AR 2000 Maximum length sequence brainstem auditory evoked responses in term neonates who have perinatal hypoxiaischaemia. Pediatr Res 48:639-645

16. Jiang ZD, Brosi DM, Wang J, Xu X, Chen GQ, Shao XM, Wilkinson AR 2003 Time course of brainstem pathophysiology during first month in term infants after perinatal asphyxia, revealed by MLS BAER latencies and intervals. Pediatr Res 54:680-687

17. Jiang ZD, Brosi DM, Wang J, Wilkinson AR 2004 Brainstem auditory-evoked responses to different rates of clicks in small-for-gestational age preterm infants at term. Acta Paediatr 93:76-81

18. Hack M, Taylor HG 2000 Perinatal brain injury in preterm infants and later neurobehavioral function. JAMA 284:1973-1974

19. Jiang ZD 1999 Outcome of brainstem auditory electrophysiology in children who survived purulent meningitis. Ann Otol Rhinol Laryngol 108:429-434

20. Jiang ZD, Yin R, Shao XM, Wilkinson AR 2004 Brainstem auditory impairment during the neonatal period in infants after asphyxia: dynamic changes in brain-stem auditory evoked responses to different rates. Clin Neurophysiol 115:1605-1615 